\title{
Crystallographic Structure Study of a Japanese Sword Masamitsu made in the 1969 using Pulsed Neutron Imaging
}

\author{
Kazuma Ohmae ${ }^{1, a^{*}}$, Yoshiaki Kiyanagi ${ }^{1, b}$, Hirotaka Sato ${ }^{2, c}$, Kenichi Oikawa,d, \\ Anh Hoang Pham ${ }^{4, e}$, Kenichi Watanabe ${ }^{1, f}$, Yoshihiro Matsumoto ${ }^{5, g}$, \\ Takenao Shinohara ${ }^{3, \mathrm{~h}}$, Tetsuya Kai ${ }^{3, \mathrm{i}}$, Stefanus Harjo ${ }^{3, \mathrm{j}}$, Masato Ohnuma ${ }^{2, \mathrm{k}}$, \\ Shigekazu Morito ${ }^{4,1}$, Takuya Ohba ${ }^{4, m}$, Akira Uritani $^{1, n}$, Masakazu Ito ${ }^{6,0}$ \\ ${ }^{1}$ Graduate School of Engineering, Nagoya University, Aichi, 464-8603, Japan \\ ${ }^{2}$ Faculty of Engineering, Hokkaido University, Hokkaido 060-8628, Japan \\ ${ }^{3}$ J-PARC Center, Japan Atomic Energy Agency, Ibaraki, 319-1195, Japan \\ ${ }^{4}$ Interdisciplinary Faculty of Science and Engineering, Shimane University, Shimane 690-8504, \\ Japan \\ ${ }^{5}$ Comprehensive Research Organization for Science and Society, Ibaraki 319-1106, Japan \\ ${ }^{6}$ WAKOU MUSEUM, Shimane 692-0011, Japan \\ aoomae.kazuma@f.mbox.nagoya-u.ac.jp, bkiyanagi@phi.phys.nagoya-u.ac.jp, \\ ch.sato@eng.hokudai.ac.jp, ${ }^{d}$ kenichi.oikawa@j-parc.jp, eanhpham@riko.shimane-u.ac.jp, ${ }^{f} k-$ \\ watanabe@energy.nagoya-u.ac.jp, 9y_matsumoto@cross.or.jp, "takenao.shinohara@j-parc.jp, \\ itetsuya.kai@j-parc.jp, jstefanus.harjo@j-parc.jp, kohnuma.masato@eng.hokudai.ac.jp, \\ Imosh@riko.shimane-u.ac.jp, mohba@riko.shimane-u.ac.jp, nuritani@energy.nagoya-u.ac.jp, \\ ${ }^{0}$ masakazu_itoh@cup.ocn.ne.jp
}

Keywords: Japanese Sword, Masamitsu, Martensite, Crystallographic Structure, Pulsed Neutron Imaging, Bragg-Edge Transmission, RITS Code

Abstract. Energy-resolved neutron transmission imaging technique using an accelerator-driven pulsed neutron source and a time-resolved two-dimensional detector can obtain the Bragg-edge transmission spectrum at each pixel. In this technique, crystallographic information can be visualized over a wide area of a sample. We used this method to investigate the crystallographic information of a modern Japanese sword produced by Masamitsu in 1969. As a result, shifting and broadening of the (110) Bragg-edge was confirmed at the cutting-edge side. It is evidence that the martensite phase exists at the cutting-edge side as a result of the quenching process. By comparing with results of the old Japanese swords, it is found that the martensite region in the modern Japanese sword is wider or deeper than one in the old swords. In addition, we can see strongly preferred orientation of the crystal grains at the boundary between the martensite phase on the cutting-edge side and the ferrite phase on the back-edge side.

\section{Introduction}

The metallographic characteristics are essential information to clarify making processes of the Japanese swords, because these processes were not documented well in the past. The Japanese swords have been produced in various eras and areas since ancient times. In order to discuss characteristics of the Japanese swords depending on areas and eras, we need to analyze many Japanese swords. It is necessary to analyze them non-destructively because the old Japanese swords have historical value. Neutron imaging is a powerful tool to study metallic cultural heritages because of non-destructive, high penetrating power and capability to get crystallographic information [1-2]. By analyzing the position dependent Bragg-edge spectra, 
quantitative visualization of the crystallographic information of the Japanese swords can be achieved [3].

We plan to investigate historical Japanese swords. The comparison of modern and historical swards will lead to a better understanding of the various crystallographic characteristics. In this work, we investigated crystallographic information of the modern Japanese sword made by Masamitsu in 1969.

\section{Experimental procedure}

In this work, we measured a modern Japanese sword in order to compare with the old Japanese swords. The Japanese swords are generally made of a carbon steel and was quenched and tempered.

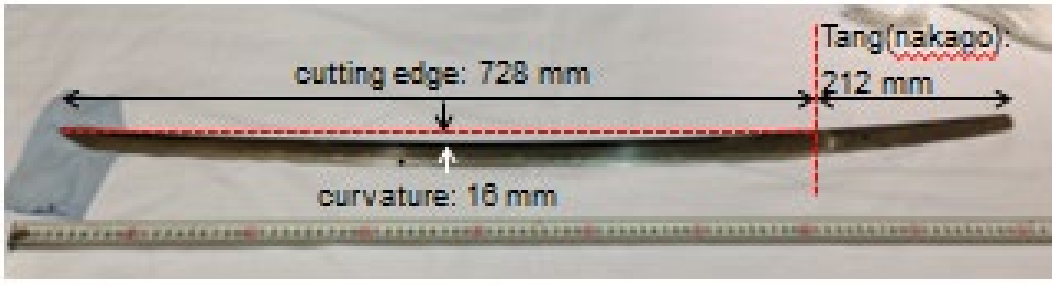

Fig. 1 A photograph of the Masamitsu sword (1969). Overview of the modern Japanese sword made by Masamitsu in 1969 is shown in Fig. 1. This experiment was performed at BL22 RADEN in JPARC MLF [4]. The proton beam power in the present work was $150 \mathrm{~kW}$. The neutron moderator was a decoupled-type supercritical para- $\mathrm{H}_{2}$ moderator. As a time-resolved two-dimensional detector, we adopted a neutron sensitive gas electron multiplier (n-GEM) detector [5] with a $0.8 \times 0.8 \mathrm{~mm}^{2}$ pixel resolution and a $10.24 \times 10.24 \mathrm{~cm}^{2}$ detection area (128ch $\times 128 \mathrm{ch})$. The sword and a

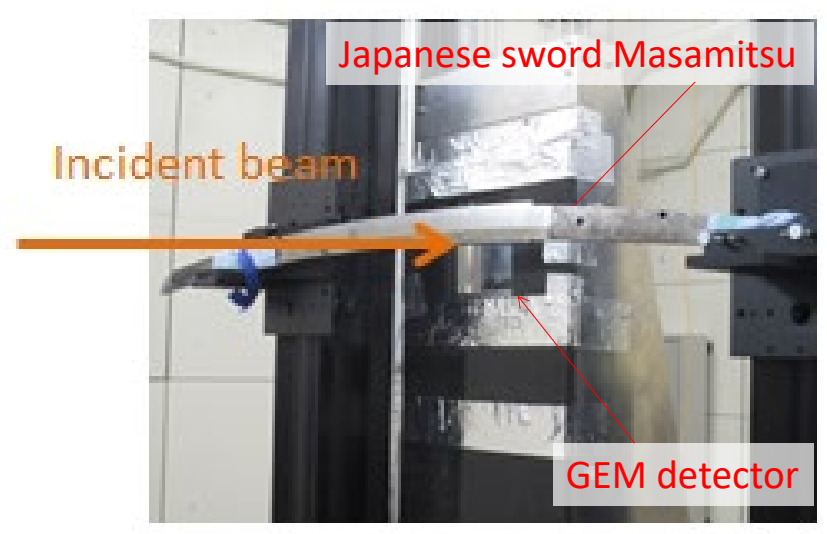

Fig.2 The experimental setup of the present work at RADEN. detector were placed at $24 \mathrm{~m}$ from the neutron source. The collimator ratio L/D was 1000 since a pinhole placed at $8 \mathrm{~m}$ from the neutron source was set in the diameter of 15 $\mathrm{mm}$. The wavelength resolution $\Delta \lambda / \lambda$ at $\lambda=0.4 \mathrm{~nm}$ was $0.2 \%$.

The experimental setup used is shown in Fig. 2. As shown in Fig. 3, the Japanese sword was measured in three different areas, Tang, Middle and Tip areas. Measurement time was 7.2 hours in the Tang area, 8.6 hours in the Middle area, 7.2 hours in the Tip area and 6 hours for the direct beam.

(a)

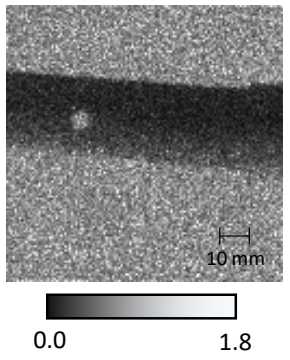

(b)

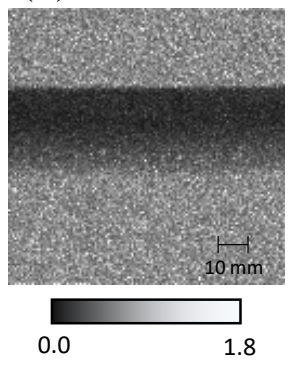

(c)

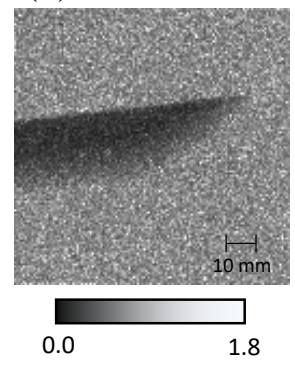

Fig. 3 Transmission images of the (a)Tang area, (b)Middle area and

(c) Tip area. 


\section{Bragg-edge analysis}

By using a time-resolved two-dimensional neutron detector at a pulsed neutron source, we can acquire an energy- or wavelengthdependent transmission spectrum at each pixel in the detector with the time-of-flight (TOF) technique. From the neutron transmission spectra of a polycrystalline metallic material, the Bragg-edge structures shown in Fig. 4 can be obtained. Since the Bragg-edge shape appears as a result of neutron diffraction in a polycrystalline or a powder sample, this spectrum contains crystal structure information of the sample. We can extract some information, such as the

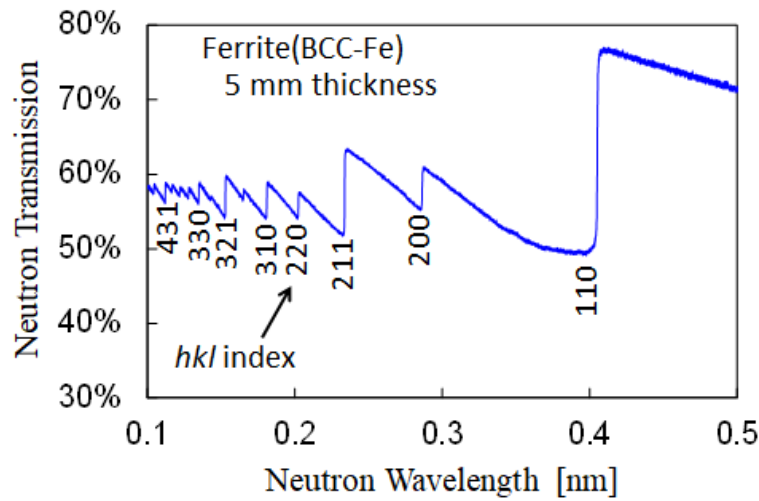

Fig.4 The Bragg-edge transmission spectrumof a ferrite plate (BCC-Fe, $5 \mathrm{~mm}$ projected atomic number density, lattice constant, preferential orientation and crystallite size, from the Bragg edge spectrum.

The Bragg-edge spectra were analyzed by the neutron Bragg-edge analysis code RITS [6]. The RITS code has two analysis modes, a single-Bragg-edge analysis mode and a Rietveld-type (wide wavelength bandwidth) analysis mode. The single-Bragg-edge analysis mode was used to extract the precise Bragg-edge position and the Bragg-edge broadening by fitting the experimental spectrum to Jorgensen function. The Rietveld-type analysis, in which the whole Bragg-edge spectrum shape is determined through the non-linear least square fitting procedure, was used to extract the projected atomic number density, preferential orientation and crystallite size [6]. In order to improve data statistics, neutron counts in $2 \times 2$ pixels $\left(1.6 \times 1.6 \mathrm{~mm}^{2}\right)$ were summed up into one pixel. As a result, $64 \times 64$ pixels two-dimensional Bragg-edge spectra were obtained. We can visualize the crystallographic information of the modern Japanese sword (Masamitsu, 1969) by mapping those refined parameters.

\section{Results and Discussion \\ Single-Bragg-edge analysis}

In the Japanese sword that is generally made of a carbon steel, the cutting-edge side is usually quenched to make the martensite phase to improve the hardness. The martensite is formed in carbon steels by rapid cooling or quenching of the austenite of iron, which allows for higher carbon content than the ferrite. In the quenching process, carbon atoms have no time to diffuse out of the crystal structure. Consequently, the face-centered cubic austenite transforms into a strained body-centered tetragonal martensite phase. The crystal lattice of martensite is distorted from the pure body-centered cubic structure. A clear difference in the Bragg-edge-position shift $\left(\mathrm{d}_{110}\right)$ and its broadening $\left(\mathrm{w}_{110}\right)$ between the ferrite and the martensite phase can be seen in the Bragg-edge spectra [7]. In general, for the martensite phase, the Bragg-edge position is shifted to the longer wavelength side and the width is broader than that of the ferrite phase. This tendency was also confirmed in the Japanese sword.

Fig. 5 (a-c) and Fig. 6 (a-c) shows the 2D maps of the lattice spacing $d_{110}$ and the Bragg-edge broadening $\mathrm{w}_{110}$. Because the Bragg edge of the $\{110\}$ crystal lattice plane is the largest, it was used in this analysis. As shown in Fig. 5 (a-c), $d_{110}$ increases near the cutting-edge. However, the boundary region between the martensite and the ferrite phase seems to have slightly smaller $d_{110}$ values compared with the normal ferrite region. Fig. 6 (a-c) shows that $\mathrm{w}_{110}$ also increases on the cutting-edge side. The width of a region showing large $d_{110}$ and $w_{110}$ is about $8 \mathrm{~mm}$. The wider region on the cutting-edge side was quenched compared with the old Japanese swords [8,9]. It is 
considered that quenching condition of the modern Japanese swords might differ from that of the old sword. Almost the whole region in Fig. 5 (a) shows non-martensite feature. This is because the Tang part was not quenched.

(a)

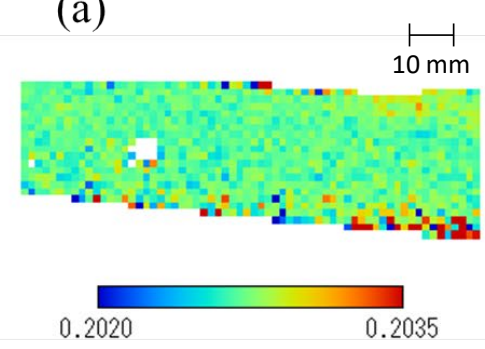

(b)

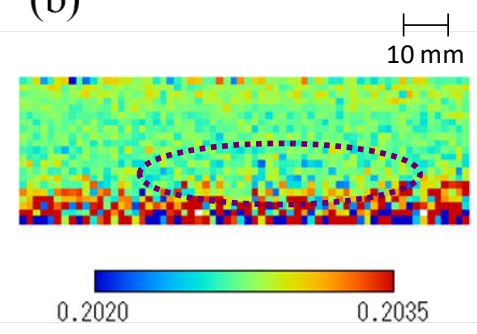

(c)

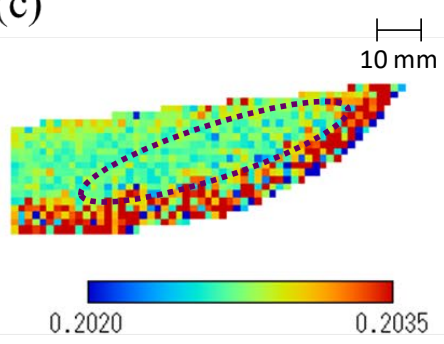

Lattice spacing $\mathrm{d}_{110}[\mathrm{~nm}]$

Fig.5 2D maps of the lattice spacing $d_{110}$ of the Masamitsu sword. (a)Tang area, (b)Middle area and (c) Tip area. The regions surrounded by dashed circles show lower values of the spacing $d_{110}$.

(a)

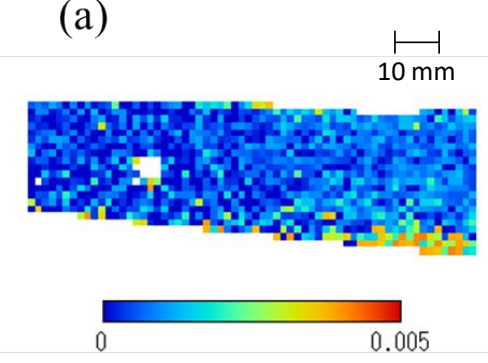

(b)

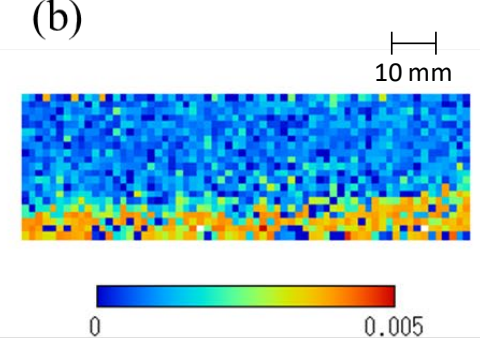

Edge broadening $\mathrm{w}_{110}[\mathrm{~nm}]$ (c)

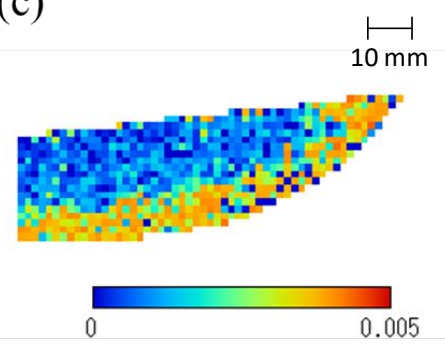

Fig.6 2D maps of the edge broadening $w_{110}$ of the Masamitsu sword.

\section{Rietveld-type analysis}

Fig. 7 (a-c) shows the 2D maps of the projected atomic number density $(\rho t)$ of the Masamitsu. The projected atomic number density corresponds to the thickness of the sample. It shows that the physical shape of the Masamitsu changes smoothly. This map reflects the actual sword thickness.

Fig. 8 (a-c) shows the 2D maps of the crystallite size (s) of the Masamitsu. The crystallite size is quantified by Sabine's primary extinction function [6]. This result shows that the crystallite size is sufficiently small over the whole region in the sword. It is considered that coarsening of crystal grain was not occurred in the heating processes.

Fig. 9 (a-c) shows the 2D maps of the preferred-orientation parameter $(r)$. The parameter $r$ is quantified by March-Dollase function [6]. If the orientation distribution of crystal grains is completely random, $r$ should be 1 . If the preferred-orientation vector $<\mathrm{hkl}>$ is parallel to the neutron beam axis, $r$ becomes less than 1 . On the other hand, if the $<\mathrm{hkl}>$ plane is perpendicular to the beam axis, $r$ becomes greater than 1 . In this case, the $<210>$ vector showed the best fitting results. Fig. 9 (c) shows that $r$ is nearly 1 for the whole region at the Tip area. However, the boundary between the martensite and ferrite region shows greater $r$ values in the Tang and Middle areas (Fig. 9 (a-b)). In order to discuss the difference in preferred-orientation feature between the tip and other regions, we have to investigate in more detail. The spatial distribution of the preferred-orientation might suggest some differences in sword fabrication processes. 

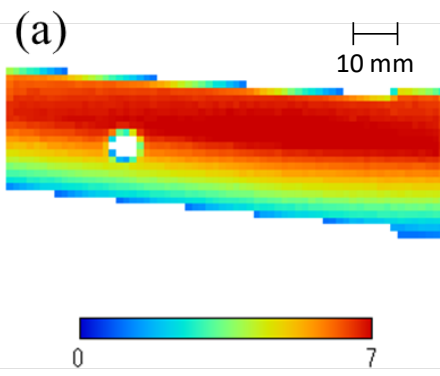

(b)
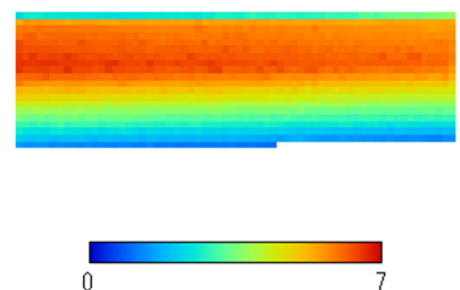

(c) $\stackrel{\vdash}{10 \mathrm{~mm}}$

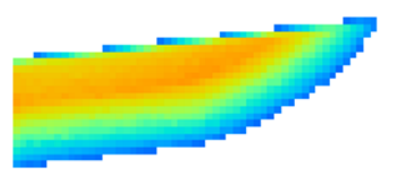

0

Projected(Area) atomic number density $\left[\times 10^{22} \mathrm{~cm}^{-2}\right]$

Fig. $72 D$ maps of the projected atomic number density $\rho$ of the Masamitsu sword.
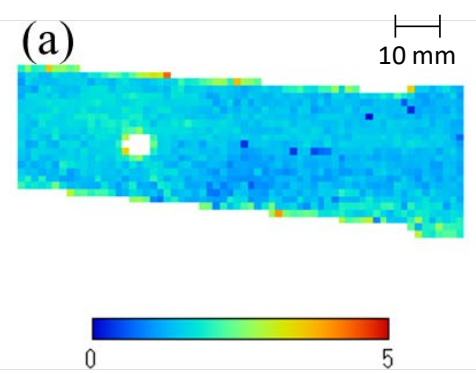

(b)
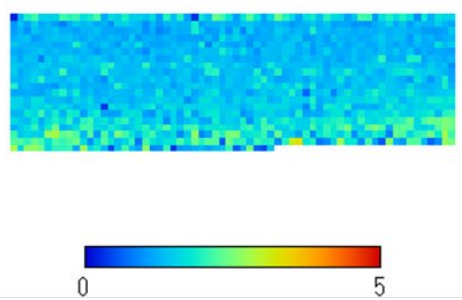

(c)
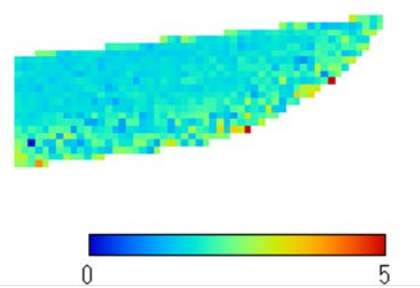

Crystallite size $[\mu \mathrm{m}]$

Fig.8 2D maps of the crystallite size s of the Masamitsu sword.

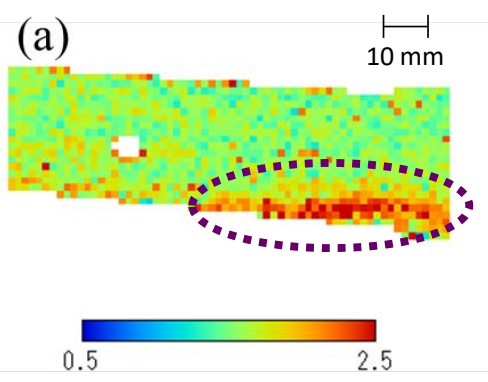

(b)

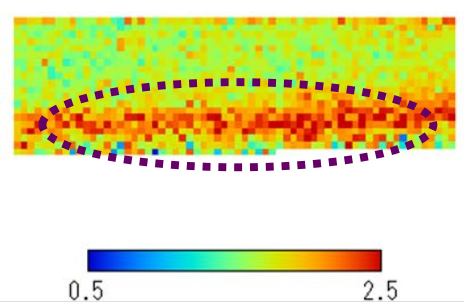

March-Dollase coefficient (c) $\stackrel{\longmapsto}{10 \mathrm{~mm}}$
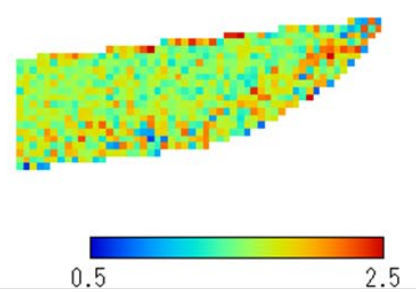

Fig.9 2D maps of the preferred-orientation parameter $r$ of the Masamitsu sword. The regions surrounded by dashed circles show higher values of the preferred-orientation parameter $r$.

\section{Conclusions}

We conducted the neutron Bragg-edge imaging to extract crystallographic information of a modern Japanese sword Masamitsu made in 1969. Valuable information of the crystallographic microstructure was revealed as follows:

(1) The cutting-edge side has larger lattice spacing and broader Bragg-edge width than that of the other side. This means that the martensite phase was created in the cutting-edge region. The width of the martensite region in the modern Japanese sword is wider than that of the old ones. It is considered that quenching condition of the modern Japanese swords might differ from that of the old sword. 
(2) Crystallite size seems to be well small over the whole region in the sword. It is considered that coarsening of crystal grain was not occurred in the heating processes.

(3) At the Middle and Tang areas, the preferred orientation near the boundary between the martensite and ferrite phase seems to be stronger than that of the other part. The spatial distribution of the preferred-orientation might suggest some differences in sword fabrication processes.

This work is step towards a better understanding of fabrication processes of historical Japanese swords. Some differences between the modern and old Japanese swords were confirmed. We will further investigate the detailed information on the micro-structural properties of the Japanese swords.

\section{Acknowledgements}

The neutron experiments at the J-PARC MLF BL22 "RADEN" performed under Proposal No. 2017A0099. This work partially includes the result of 'Collaborative Important Researches' organized by JAEA, QST and U. Tokyo.

\section{References}

[1] F. Salvemini, F. Grazzi, S. Peetermans, et al., Quantitative characterization of Japanese ancient swords through energy-resolved neutron imaging. J. Analytical Atomic Spectrometry 2012, 27, 1494-1501. https://doi.org/10.1039/c2ja30035d

[2] A. Fedrigo, M. Strobl, A.R. Williams, et al., Neutron imaging study of 'pattern-welded' swords from the Viking Age, Archaeol. Anthropol. Sci., 2018, 10, 1249 - 1263.

https://doi.org/10.1007/s12520-016-0454-5

[3] Y.Shiota, H. Hasemi, Y. Kiyanagi, Crystallographic analysis of a Japanese sword by using Bragg edge transmission spectroscopy, Phys. Procedia 2017, 88, 128-133.

https://doi.org/10.1016/j.phpro.2017.06.017

[4] T. Shinohara, T. Kai, K. Oikawa, et al., Final design of the Energy-Resolved Neutron Imaging System “RADEN” at J-PARC. J. Phys. Conf. Ser. 2016, 746, 012007. https://doi.org/10.1088/1742-6596/746/1/012007

[5] M. Shoji, S. Uno, T. Uchida, et al., Development of GEM-based detector for thermal neutron, J. Inst., 2012, 7, C05003. https://doi.org/10.1088/1748-0221/7/05/C05003

[6] H. Sato, T. Kamiyama, Y. Kiyanagi, A Rietveld-type analysis code for pulsed neutron Bragg-edge transmission imaging and quantitative evaluation of texture and microstructure of a welded $\alpha$-iron plate. Mater. Trans. 2011, 52, 1294-1302.

https://doi.org/10.2320/matertrans.M2010328

[7] H. Sato, T. Sato, Y. Shiota, et al., Relation between Vickers Hardness and Bragg-Edge Broadening in Quenched Steel Rods Observed by Pulsed Neutron Transmission Imaging. Mater. Trans. 2015, 56, 1147-1152. https://doi.org/10.2320/matertrans.M2015049

[8] K. Oikawa, Y. Kiyanagi, H. Sato, et al., Pulsed neutron imaging based crystallographic structure study of a Japanese sword made by Sukemasa in the Muromachi period, Mater. Res. Proc. (this proceedings).

[9] H. Sato, Y. Kiyanagi, K. Oikawa, et al., Crystallographic microstructure study of a Japanese sword made by Noritsuna in the Muromachi period by pulsed neutron Bragg-edge transmission imaging, Mater. Res. Proc. (this proceedings). 